\title{
A self-contained mental health outpost: An automated approach to prevention
}

\author{
WILLIAM G. HARRIS, ALEXANDER A. ESCHBACH, and JAMES H. JOHNSON \\ Illinois Institute of Technology, Chicago, Illinois 60616
}

\begin{abstract}
This paper describes the application of a microcomputer system as a mental health outpost. The outpost contains a psychological screening inventory, a relaxation training and educational session, and a consumer-satisfaction questionnaire. A study to assess the validity of the decision making algorithm of the mental health outpost is reported. The implications of an automated delivery system for first-level preventive and promotional mental health services are discussed.
\end{abstract}

In the last 2 decades, the application of computer technology to mental health care delivery has grown substantially. During this period, three major efforts to utilize computer technology to facilitate the delivery of mental health services emerged (Johnson, Giannetti, \& Williams, 1976). The first major effort, which began in the early 1960s, focused on the development of automated patient data systems. Prime examples of this early application of computers were the Multi-State Information System (MSIS; Laska, Logemann, Honigfeld, Weinstein, \& Banks, 1972) and the Missouri Standard System of Psychiatry (SSOP; Sletten \& Ulett, 1972). These systems provided an efficient method for handling patient record keeping. The second effort also began in the early 1960s. It centered on the development of automated clinical techniques. Psychological tests (Pearson, Swenson, Rome, Mataya, \& Brannick, 1965), psychiatric techniques (Spitzer \& Endicott, 1974; Spitzer, Fleis, Endicott, \& Cohen, 1967), social histories (Johnson \& Williams, 1975), and nursing techniques (Stroebel \& Glueck, 1970) were some of the clinical areas that proved to be amenable to automation.

Although considerable progress had been made by the 1970s in applying computer technology to the delivery of mental health care, there was still very little consensus among mental health professionals that this union was beneficial or desirable (Johnson, 1978). The opposition of mental health professionals to the intrusion of computer technology on the clinician's domain prompted the third and most recent effort to demonstrate the utility of computer technology in providing mental health services. This third endeavor has aimed to develop "need-based" or "interventionally relevant" computer systems to deliver mental health care. Based on this perspective, specialized computer systems have been designed to meet specific mental health needs that were not being effectively handled by traditional mental health service delivery approaches.

Increased interest in the application of interventionally relevant delivery methods in mental health was further spurred by the advent of microprocessing computer systems (Johnson, 1978). These computer systems allow the individual to interact directly with the computer at low cost. This means that information obtained on an individual (e.g., by psychological assessment) can be readily processed and made available at various decision points during treatment. Studies of client satisfaction with on-line delivery of certain mental health services show consumer approval to be relatively high (Klingler, Johnson, \& Williams, 1976). There are also indications that steady but gradual support for interventionally relevant mental systems is occurring among clinicians (Johnson, 1978). Moreover, the results of evaluation studies indicate that such specialized computer systems do tend to improve the performance of mental health facilities in delivering specific services (Klingler, Johnson, \& Williams, 1976).

Although the use of need-based computer systems has increased in recent years (Evenson, Altman, Cho, \& Sletten, 1974; Linkenhoker \& McCarron, 1974;Williams, Johnson, \& Bliss, 1975), these specialized mental health computer systems are not receiving widespread use, and the multipurpose potential of these systems in mental health delivery is still a fairly untapped area.

One area of mental health care that has been difficult to adequately service has been the delivery of primary prevention and promotional mental health services. Many proponents of these front-line mental health services (Cowen, 1977; Henderson, 1975; Saunders, 1979) are in agreement with The President's Commission on Mental Health: The Final Report (1978) that efforts to provide these services have usually been "unstructured, unfocused, and uncoordinated" (p. 53). Nevertheless, the aims of front-line preventive and promotional services are to strengthen the ability of people to cope with the adjustive demands of everyday life and to assist groups of individuals in gaining greater meaning from their lives and from the social environment (McPheeters, 1976). First-level mental health activities service the mass rather than the individual and are therefore educational and informational in nature. Although there is agreement among mental health 
professionals that primary prevention and promotional mental health services are a necessary line of care, insufficient allocation of resources in this area hinders attempts of mental health practitioners to establish effective service delivery strategies. There is, however, a need to increase the performance of mental health units to deliver front-line services.

The purpose of this paper is to describe: (1) the development of a self-contained preventive and promotional mental health system, and (2) a study to test the reliability of the system's decision making capabilities. It is hoped that this study will further demonstrate the potential application of computer technology to deliver specific front-line mental health services.

The functional operation of this self-contained on-line system can be briefly described. An individual is seated in front of a microcomputer console and a CRT screen. On the CRT screen, the statement "Press ' $\mathrm{X}$ ' and the return key to learn how to promote your own mental health" is displayed. Once the person carries out this command, a description of the project appears on the screen for him/her to read. The individual is then instructed to respond to a list of demographic questions. The obtained demographic information consists of ethnic background, sex, age, marital status, education, and other related data. After completing the demographic survey, the individual is administered the Psychological Screening Inventory (PSI; Lanyon, 1973) on-line. Since the system operates in a real-time mode, the PSI data for each individual are immediately scored. The PSI results are compared with prespecified rules, and a decision about the appropriateness of services for the individual is made.

At the present stage of development, a decision of direct service means that the assessment shows that the person can benefit from the automated relaxation and stress-reduction program. This program describes the nature of stress and ways of managing stress. The individual is informed that he/she will be given a method of relaxation that, with regular use, can assist in the reduction and management of stress. This information is followed by the administration of a four-item truefalse quiz. The quiz provides the person with an indication of how well he/she understands the material. One or more incorrect responses causes the statement "Would you like to review this material again?" to be displayed on the CRT. If the response of the trainee is "yes," the information is returned to the screen for his/her perusal. If the response is "no," the next phrase of the relaxation training is introduced.

At this point in training, the person is given a list of eight basic rules for effective relaxation training. The rules cover common concerns, such as body posture, rate of practice, the importance of casual concentration, and other pertinent information. The trainee is also quizzed on his/her grasp of the basic relaxation rules, and the correct answers are made immediately available to the trainee through the CRT. The trainee can, if necessary, review the basic rules before proceeding to the next section of the relaxation training program.

In this section, the individual is given two preliminary relaxation exercises. Both preliminary exercises and subsequent exercises were adapted from Jencks' (1973) manual for J. H. Schultz' autogenic relaxation training. The first warm-up exercise is deep breathing. In the second exercise, the individual is instructed to develop a mental image that is pleasant and relaxing. When a person finds it difficult to form a mental image, a vivid description of an outdoor scene that many people have described as peaceful and tranquil is presented through the CRT. If the person is still unable to form a relaxing mental image, the computer system, through its video display, reassures the person that mental imagery, although useful, is not essential for learning effective relaxation methods.

The trainee is then given a description of the first relaxation exercise. On reading the description, the individual is put through a practice period. The amount of time for the practice session is controlled by using the audio signal on the terminal to terminate the practice period. Additional information that explains broader usage of the first exercise is also shared with the trainee.

The format for presenting the other four relaxation exercises is similar. The presentation of the second and fifth exercises is followed by a brief test of important points in relaxation training. Completion of the fifth exercise and the test prompts the system to print (for the trainee's use) an outline of the basic rules and relaxation instructions. This printed outline provides the trainee with information that can be used when he/she practices off-line. Before terminating his/her interaction with the microcomputer system, the trainee is administered a consumer-satisfaction questionnaire (CSQ). The results of the questionnaire will be used to measure the attitude of consumers to this automated service and to determine the type of modifications that are needed in the system's design. A statement emphasizing the research status of this project precedes the command that the system's interaction with the trainee has ended.

The system configuration includes a DEC 11/03 microcomputer with $32 \mathrm{~K}$ memory, a VT100 CRT, a Diablo printer, and two DEC floppy-disk drives. This equipment is inexpensive and relatively maintenance free. Another advantage of this equipment is its small size and portability.

All programming was completed in multiuser BASIC. A major reason for using multiuser BASIC was its exportability. The software specifications were designed to allow for the inclusion of additional mental health services. The addition of new services can be done by simply using the chain directive of the multiuser BASIC programming language.

In this self-contained station, the mental health assessment tool (PSI), the decision making device, the relaxation training program, and the CSQ are stored on 
one floppy disk. Subject data files include demographic information, PSI raw data, PSI scale totals for Lanyon's (1973) five original scales and Overall's (1974) three factors, the on-line service decision, and the CSQ responses. Although each of the services (e.g., the assessment, the decision, and the training session) in the mental health station is independent in functional operations, each service does interface with the other services and with the system flow.

Since the usefulness of this mental health station depends largely on the trustworthiness of its decision making algorithm, we decided to compare algorithmgenerated decisions with those of a well trained clinician to ascertain the validity of the algorithm. Forty-eight subjects were recruited from two undergraduate psychology courses at the Illinois Institute of Technology. The sample consisted of 33 males and 15 females. The mean age of the subjects was 22.75 years $(S D=4.25)$. The instrumentation was the PSI (Lanyon, 1973), a screening device that consists of five scale; alienation, social nonconformity, discomfort, expression, and defensiveness. The inventory is used to measure level of social adaptation and psychological well-being. Decision rules specifying when relaxation training was appropriate were generated using Kleinmuntz' (1963) method of clinician "thinking aloud." The clinician in this study was given a set of PSI profiles. The profiles were sorted into "adjusted" and "maladjusted" categories. The adjusted profiles were then sorted into relaxation training and no relaxation training categories. Based on these two sorts, decision rules for the on-line decision making mechanism of the mental health outpost were developed.

To test the validity of the decision making algorithm, PSI test score data for the 48 subjects were scored on-line. The algorithm then used this information to make on-line decisions about the appropriateness of relaxation training for each subject. These computerassisted decisions were then compared with those of an experienced mental health professional who was familiar with the use of the PSI and relaxation training methods with college populations. The kappa technique (Cohen, 1960) for measuring nominal agreement was used to compute interjudge reliability between the computer and the mental health professional. $\mathrm{A} \mathrm{k}=.64$ for all the observed decisions for the 48 subjects was obtained. The result was significantly greater than zero $(\mathrm{p}<.001)$. This finding suggests that the algorithm is able to make a valid decision about the suitability of computerized relaxation training for individuals interacting with the on-line mental health outpost.

Although this study demonstrates that the algorithm can make a valid decision on the appropriateness of relaxation training, such a finding can only be viewed as preliminary. In fact, we are now conducting a study to refine the decision rules using more psychometrically robust procedures. This refinement will further enhance the accuracy of the algorithm, especially when other mental health services are contained in the outpost.
Also, we are presently evaluating consumer satisfaction with the on-line relaxation session. The individual is administered an on-line questionnaire that is designed to measure his/her degree of satisfaction with the automated mental health services. After a period of 4 weeks has elapsed, a second administration of the CSQ is conducted. Comparison of the attitude of individuals immediately following the delivery of services and 4 weeks later will determine how these individuals actually perceive the effectiveness of the on-line mental health services. Partial results indicate that consumers are satisfied with the on-line relaxation program. Consumers report that they find the computerized relaxation training instructions understandable and the information immediately usable.

Although these findings are encouraging, additional computer-assisted mental health services such as assertion-training, referral information, and other activities amenable to a computer medium are needed. Without developing multiple service capabilities, an automated self-contained mental health station for delivering front-line mental health services would not be financially feasible or interventionally relevant.

This automated mental health service system does have certain limitations. By design, the services are aimed at the education of the masses rather than the psychological restoration of the individual. It is important that these automated services are not construed as psychotherapy or some other mental health activity that is not aimed at first-level prevention. Although this is a self-contained station, it is nevertheless viewed as functioning within a larger mental health system rather than as an insular outpost. It is conceptually and operationally amiss to view this station as a separate mental health delivery entity. Implicit in the notion of interventionally relevant applications of computer technology is a general systems theory approach to mental health care delivery. This means that the lines of service care delivery and their interrelationships are expected to be understood from the broader systems view.

Although the research status of this mental health station makes the present equipment configuration adequate, the eventual demonstration of this delivery approach in a community setting will force equipment modifications. For example, the placement of mental health outposts throughout a circumscribed community would require more advanced hardware and software to handle multiple user sites. Hard disk drives and hard disks would replace the present floppy-disk units. The efficient delivery of mental health services is a major attraction of this system. Therefore, the equipment used to deliver these services must be able to meet this challenge.

This study describes the functional, equipment, and systems specifications of an interventionally relevant application of microcomputers to the delivery of both preventive and promotional mental health services. An on-line psychological screening, service decision, and 
relaxation training program is described as part of a self-contained mental health station. Although the use of computers to enhance delivery of mental health services in traditional settings is now well documented, we are not aware of any attempts to use a computer medium to deliver first-level mental health services in nontraditional mental health settings. It is argued that computers can improve delivery of these services by making them more accessible and readily usable. Firstlevel prevention and promotional services are amenable to an automated medium, as is shown with the adaptation of autogenic relaxation techniques for computer application. Although we are presently conducting a more detailed evaluation of this system that includes a broader service selection range, our present work suggests that computer technology can eventually serve an important function in helping to decentralize front-line mental health services and in bringing these services to more people.

\section{REFERENCES}

Cohen, J. A coefficient of agreement for nominal scales. Educational and Psychological Measurement, 1960, 37-46.

Cowen, E. Baby-steps toward primary prevention. American Journal of Community Psychology, 1977, 5, 1-22.

Evenson, R. C., Altman, H., Cho, D. W., \& Sletten, I. W. The relationship of diagnosis and target symptoms to psychotropic drug assignment. Comprehensive Psychiatry, 1974, 15, 173-178.

Henderson, J. Object relations and a new social psychiatry: The illusion of primary prevention. Bulletin of the Menninger Clinic, 1975, 39, 233-245.

JEncks, B. Exercise manual for J. $\boldsymbol{H}$. Schultz's standard autogenic training. Salt Lake City, Utah: American Society of Clinical Hypnosis, 1973.

Jounson, J. H. Computers in mental health: Where are we now? Proceedings: The Second Annual Symposium on Computer Application in Medical Care, 1978, 104-108.

Johnson, J. H., Giannetti, R. A., \& Williams, T. A. Computers in mental health: A review of the evolution toward interventionally relevant on-line processing. Behavior Research Methods \& Instrumentation, 1976, 8, 83-91.
Johnson, J. H., \& Williams, T. A. The use of on-line computer technology in a mental health admitting system. American Psychologist, 1975, 30, 388-390.

KLEInMUnTz, B. MMPI decision rules for the identification of college maladjustment: A digital computer approach. Psychological Monograph, 1963, 77(14, Whole No. 477).

Klingler, D. E., Johnson, J. H., \& Williams, T. A. Strategies in the evaluation of an on-line computer-assisted unit for intake assessment of mental health patients. Behavior Research Methods \& Instrumentation, 1976, 8, 95-100.

Lanyon, R. I. Psychological screening inventory: Manual. Port Huron, Mich: Research Psychologists Press, 1973.

Laska, E, Logemann, G., Honigfeld, G., Weinstein, A., \& BANR, R. The multi-state information system. Evaluation, 1972, 1, 66-71.

Linkenhoker, D. D., \& McCarron, L. T. Computerized assessment programming (CAP): A prescriptive strategy for delinquents. Behavioral Science, 1974, 19, 254-259.

McPheEters, H. L. Primary prevention and health promotion in mental health. Preventive Medicine, 1976, 5, 187-198.

Overall, J. E. Validity of the Psychological Screening Inventory for psychiatric screening. Journal of Consulting and Clinical Psychology, 1974, 5, 717-719.

Pearson, J. S., Swenson, W. M., Rome, H. P., Mataya, P., \& Brannick, T. L. Development of a computer system for scoring and interpretation of Minnesota Multiphasic Personality Inventories in a medical clinic. Annals of the New York Academy of Sciences, 1965, 126, 682-692.

The President's Commission on Mental Health: The final report (Vol. 1). Washington, D.C: U.S. Government Printing Office, 1978.

SAunders, S. Primary prevention from a neighborhood base: A working model. American Journal of Orthopsyschiatry, 1979, 49, 69-80.

Sletten, I. W., \& UletT, G. A. The present status of automation in a state psychiatric system. Psychiatric Annals, 1972, 2, $42-57$.

SpItzer, R. L., \& EndicotT, J. Can the computer assist clinicians in psychiatric diagnosis? American Journal of Psychiatry, 1974, 131, 523-530.

Spitzer, R. L., Fleiss, J. L., Endicott, J., \& Cohen, J. Mental status schedule: Properties of factor-analytically derived scales. Archives of General Psychiatry, 1967, 16, 479-493.

Stroebel, C. F., \& Glueck, B. C., JR. Computer derived global judgements in psychiatry. American Journal of Psychiatry, 1970, 126, 1057-1066.

Williams, T. A., Johnson, J. H., \& Bliss, E. L. A computerassisted psychiatric assessment unit. American Journal of Psychiatry, 1975, 132, 1074-1076. 